\title{
SDSS DR7 superclusters
}

\section{The catalogues ${ }^{\star}$}

\author{
L. J. Liivamägi ${ }^{1,2}$, E. Tempel ${ }^{1,3}$, and E. Saar ${ }^{1,4}$ \\ 1 Tartu Observatory, 61602 Tõravere, Estonia \\ e-mail: juhan.liivamagi@ut.ee \\ 2 Institute of Physics, University of Tartu, Tähe 4, 51010 Tartu, Estonia \\ 3 National Institute of Chemical Physics and Biophysics, Rävala 10, 10143 Tallinn, Estonia \\ ${ }^{4}$ Estonian Academy of Sciences, Kohtu 6, 10130 Tallinn, Estonia
}

Received 9 December 2010 / Accepted 22 November 2011

\begin{abstract}
We have constructed a set of supercluster catalogues for the galaxies from the SDSS survey main and luminous red galaxy (LRG) flux-limited samples. To delineate superclusters, we calculated luminosity density fields using the $B_{3}$-spline kernel of the radius of $8 h^{-1} \mathrm{Mpc}$ for the main sample and $16 h^{-1} \mathrm{Mpc}$ for the LRG sample and define regions with densities over a selected threshold as superclusters, while utilising almost the whole volume of both samples. We created two types of catalogues, one with an adaptive local threshold and a set of catalogues with different global thresholds. We describe the supercluster catalogues and their general properties. Using smoothed bootstrap, we find uncertainty estimates for the density field and use these to attribute confidence levels to the catalogue objects. We have also created a test catalogue for the galaxies from the Millennium simulation to compare the simulated and observed superclusters and to clarify the methods we use. We find that the superclusters are well-defined systems, and the properties of the superclusters of the main and LRG samples are similar. We also show that with adaptive local thresholds we get a sample of superclusters, the properties of which do not depend on their distance from the observer. The Millennium galaxy catalogue superclusters are similar to those observed.
\end{abstract}

Key words. large-scale structure of Universe - galaxies: clusters: general

\section{Introduction}

The large-scale structure of the galaxy distribution is characterised by large voids and by a complex web of galaxy filaments and clusters. Superclusters are the largest components of the cosmic web. They are collections of galaxies and galaxy clusters, with typical sizes of $20-100 h^{-1} \mathrm{Mpc}$. They can contain up to hundreds of galaxy groups and several rich clusters. The first described supercluster is the Local Supercluster (de Vaucouleurs 1953), and many other superclusters have been found and studied in our neighbourhood.

Astronomers have a long tradition of selecting galaxy clusters and groups from this web, but quantifying the overall web is a much more difficult task. This can be done in several ways, all of them computer-intensive and based on the properties of a smoothed galaxy density field. Good recent examples are the application of the multiscale morphology filter by Aragón-Calvo et al. (2010) and the Bayesian inference for the density and the subsequent classification of the web elements by Jasche et al. (2010). These articles contain an exhaustive set of references. In this approach, the different sets of web components differ mainly in their dimensionality (clusters, filaments, sheets, and voids). Another approach that has been used is to divide the observed weblike galaxy distribution into its main building blocks - superclusters. Superclusters are frequently treated in a similar way

* Catalogues are available at the CDS via anonymous ftp to cdsarc.u-strasbg.fr (130.79.128.5) or via

http://cdsarc.u-strasbg.fr/viz-bin/qcat?]/A+A/539/A80 to groups and clusters of galaxies - they are density enhancements in the overall galaxy distribution.

This approach leads to the construction of supercluster catalogues on the basis of both Abell clusters (Einasto et al. 1997, 2001) and galaxy groups (Einasto et al. 2007), using smoothed density fields. A similar method has recently been used by Costa-Duarte et al. (2011) and Luparello et al. (2011). The friends-of-friends method was used by Basilakos (2003) to compile superclusters from the SDSS sample.

Supercluster catalogues are similar to other astronomical catalogues, because while serving as a basis to describe and study classes of objects, they are also essential for further work. This includes planning observational projects, comparing different classes of astronomical objects, and comparing theory (simulations) with observations. We present here the supercluster catalogues based on the richest existing redshift survey, the SDSS DR7. These catalogues have already been used for several studies. The list includes a study of the locations of quasars within the large-scale structure delineated by galaxies (Lietzen et al. 2009), a couple of observing proposals to search for the warm-hot intergalactic medium, and a morphological study of the rich superclusters forming the Sloan Great Wall (Einasto et al. 2010). This catalogue has also been used for a preliminary identification of a Sunyaev-Zel'dovich (SZ) source seen in the early Planck mission data (Planck Collaboration et al. 2011).

The paper is organised as follows. In Sect. 2 we describe our method (beginning with the calculation of the density field), outline supercluster delineation principles, and explain how some of the more important properties of the superclusters are calculated. 
In this section we also address the errors of the density field estimates. In Sect. 3 we describe the datasets used. Supercluster properties are described in Sect. 4, where we also compare different samples. The resulting catalogue can be downloaded from: http://atmos.physic.ut.ee/ juhan/super/with a complete description in the readme files. We will also upload selected parts of the catalogues (listed in Appendix C) to the Strasbourg Astronomical Data Center (CDS).

\section{Delineating superclusters by the luminosity density field}

We define superclusters on the basis of their total density that is dominated by dark matter. Supposing that the bias (the ratio of the dark matter density to the stellar density) is approximately constant on supercluster scales, the observational counterpart for the total density is the luminosity density. We do not use clusters or groups to create the density field, as done for earlier supercluster catalogues by Einasto et al. (2003, 2007), but the full galaxy distribution. Before calculating the density field we processed the galaxy data to reduce several observational selection effects. The galaxy and group samples we used are described in Sect. 3 .

\subsection{Distance and luminosity corrections for the SDSS main sample}

The spectroscopic galaxy samples (as the SDSS) are affected by the cluster-finger redshift distortions (the fingers-of-god). To suppress the cluster-finger redshift distortions, we use the rms sizes of galaxy groups and their radial velocity dispersions from the Tago et al. (2010) galaxy group catalogue. In this catalogue, the comoving distances (see e.g. Martínez \& Saar 2002) are used for galaxies and groups, in units of $h^{-1} \mathrm{Mpc}$. For groups with three or more members, we divide the radial distances between the group galaxies and group centres ( $\left.d_{\text {group }}\right)$ by the ratio of the standard deviations $\sigma_{r} / \sigma_{v}$. This will remove the smudging of the density field by the cluster fingers. The corrected galaxy distance $d_{\text {gal }}$ is found as

$d_{\text {gal }}=d_{\text {group }}+\left(d_{\text {gal }}^{\star}-d_{\text {group }}\right) \frac{\sigma_{r}}{\sigma_{v} / H_{0}}$,

where $d_{\text {gal }}^{\star}$ is the initial distance of the galaxy, $\sigma_{r}$ the standard deviation of the projected distance in the sky from the group centre, $\sigma_{v}$ the standard deviation of the radial velocity (both in physical coordinates at the group location), and the Hubble constant $H_{0}=100 \mathrm{~h} \mathrm{~km} \mathrm{~s}^{-1} \mathrm{Mpc}^{-1}$.

We use a Cartesian grid based on the SDSS angular coordinates $\eta$ and $\lambda$, because it allows the most efficient placing of the galaxy sample cone inside a box. The galaxy coordinates are calculated as follows:

$$
\begin{aligned}
& x=-d_{\mathrm{gal}} \sin \lambda, \\
& y=d_{\mathrm{gal}} \cos \lambda \cos \eta, \\
& z=d_{\mathrm{gal}} \cos \lambda \sin \eta .
\end{aligned}
$$

To compensate for selection effects and to ensure that the reconstructed density field does not depend on the distance, we have to take the luminosities of the galaxies into account that drop out of the survey magnitude window. We follow the procedure by Tempel et al. (2011) and consider every galaxy as a visible member of a density enhancement (a group or cluster) within the visibility range at the distance of the galaxy. We estimate the amount of unobserved luminosity and weigh each galaxy as

$L_{\mathrm{gal}, \mathrm{w}}=W_{L}(d) L_{\mathrm{gal}}$,

where $L_{\text {gal }}=L_{\odot} 10^{0.4\left(M_{\odot}-M\right)}$ is the observed luminosity of a galaxy with the absolute magnitude $M$, and $M_{\odot}$ is the absolute magnitude of the Sun. The quantity $W_{L}(d)$ is the distancedependent weight factor: the ratio of the expected total luminosity to the luminosity within the visibility window:

$W_{L}(d)=\frac{\int_{0}^{\infty} L \phi(L) \mathrm{d} L}{\int_{L_{1}(d)}^{L_{2}(d)} L \phi(L) \mathrm{d} L}$,

where $L_{1,2}(d)$ are the luminosity limits corresponding to the survey magnitude limits $M_{1,2}$ at the distance $d$.

We approximate the luminosity function by a double power law:

$n(L) \mathrm{d}(L) \propto\left(L / L^{*}\right)^{\alpha}\left(1+\left(L / L^{*}\right)^{\gamma}\right)^{(\delta-\alpha) / \gamma} \mathrm{d}\left(L / L^{*}\right)$,

where $\alpha$ is the exponent at low luminosities $\left(L / L^{*}\right) \ll 1, \delta$ the exponent at high luminosities $\left(L / L^{*}\right) \gg 1, \gamma$ a parameter that determines the speed of the transition between the two power laws, and $L^{*}$ the characteristic luminosity of the transition. This form represents the bright-magnitude end of the luminosity function better than the usual Schechter function (Tempel et al. 2009).

\subsection{Luminosity corrections for the LRG sample}

Although the luminosity function of the SDSS LRGs has already been determined (Wake et al. 2006), it is difficult to calculate the luminosity weights for LRGs as we did above for the main sample. The reason is simple - the LRG sample does not have the two magnitude limits. Because of that, we find the observed comoving luminosity density $\ell(d)$ and defined the luminosity weight as its inverse:

$W_{L}(d)=\ell\left(d_{0}\right) / \ell(d)$

where $d_{0}$ is the fiducial comoving distance (taken as $435.6 h^{-1} \mathrm{Mpc}$, see Sect. 3.2).

Both these luminosity correction schemes (for the main and LRG samples) add luminosity to the observed galaxy locations, and cannot restore the real, unobserved galaxies. This evidently increases the shot noise at distances, but that is unavoidable.

\subsection{Calculation of the luminosity density field}

We describe the mathematical details for calculations for the luminosity density field in Appendix A; here we give a brief summary of the procedure. We denote the luminosity density field on a grid with $\ell_{i}$, where $\boldsymbol{i}=\left(i_{1}, i_{2}, i_{3}\right)$ are the indices of the vertices. The luminosity densities are calculated by a kernel sum:

$\ell_{i}=\frac{1}{a^{3}} \sum_{\mathrm{gal}} K^{(3)}\left(\frac{\boldsymbol{r}_{\mathrm{gal}}-\boldsymbol{r}_{\boldsymbol{i}}}{a}\right) L_{\mathrm{gal}, \mathrm{w}}$,

where $L_{\mathrm{gal}, \mathrm{w}}$ is the weighted galaxy luminosity, and $a$ the kernel scale.

We use the $B_{3}$ spline kernel $B_{3}(x / a)$ (see Appendix A) to construct the one-dimensional kernel $K^{(1)}(x / a)$, and form the three-dimensional kernel as a direct product of three onedimensional kernels. The scale $a$ can be regarded as the effective 


\section{J. Liivamägi et al.: SDSS DR7 superclusters}

radius of the kernel, and its choice is determined by the application.

As the last step before extracting superclusters, we convert densities into the units of mean density. The main purpose of this is to facilitate comparison between different density fields. For that, we construct a pixel mask that follows the sample edges. We determine the mean density as an average over all vertices inside the mask,

$\ell_{\text {mean }}=\frac{1}{N_{\text {mask }}} \sum_{i \in \text { mask }} \ell_{i}$,

where $N_{\text {mask }}$ is the number of grid vertices inside the mask. We finally normalise the density field as

$D_{i}=\frac{\ell_{i}}{\ell_{\text {mean }}}$,

for all vertices with coordinates $\boldsymbol{i}$ inside the mask. The vertices outside the mask are not used again.

We find the variances $\sigma_{\ell}^{2}$ of the density field estimates for all vertices by smoothed bootstrap, as described in Appendix B. Using that, we calculate for every grid vertex the signal-to-noise ratio

$G_{i}=\frac{\ell_{i}}{\sigma_{\ell i}}$

It is used later to estimate confidence levels of superclusters. The parameters and properties of the luminosity density fields are described in Sect. 4.1.

\subsection{Assembly of superclusters}

We define our superclusters using the luminosity density field. A conventional way is to choose a density level and to define superclusters as connected density regions above that level (see, e.g., Einasto et al. 2007; Luparello et al. 2011). For different tasks, these levels are chosen differently. Because of that, we create sets of contour surfaces for different density thresholds $D_{n}$, sampling the density range from $D_{\min }$ to $D_{\max }$ with a constant increment $\delta D$.

We use density peaks to identify superclusters (density field objects). Contiguous supercluster regions are grown pixel-wise around the peaks in the density field resulting in a marker field

$M_{n, i}=\mathrm{ID}_{\text {peak }}, \boldsymbol{i} \in\left\{\boldsymbol{i} \mid D_{\boldsymbol{i}}>D_{n}\right\}$,

where $\mathrm{ID}_{\text {peak }}$ is the density peak number. All the vertices belonging to an object are assigned the same mark value.

We start scanning the field at high densities and move on to lower density levels. Each time an object first appears, it is assigned a unique identification number that will be used for this supercluster throughout the catalogue. We keep track when an object emerges from the field and how or if it is eventually swallowed up by another density field object. If such a merger occurs, the identifier of the object with the higher peak value will be used to designate that object later on. To record the merging history of the density field objects, we order them into a tree structure encompassing all the density thresholds.

We finally assemble superclusters by distributing galaxies among the density field objects. We do this for each density threshold by correlating galaxy positions with the corresponding marker field. For the SDSS main sample we also assign galaxy groups to superclusters. If a group or a cluster is found to be in a supercluster (its centre is located inside the supercluster contour), all its member galaxies automatically also belong to the same supercluster. We also implement a lower limit of $(a / 2)^{3}$ for a volume of a supercluster, where $a$ is the smoothing scale, in order to remove small spurious density field objects that include no galaxies.

\subsection{Selection of density thresholds}

With the multitude of available thresholds comes the question which is the "correct" one? Just as there is no clear-cut definition for superclusters, there is also no single answer for this. We offer two possibilities for tackling this problem. The first one is the conventional way of choosing a fixed density level, as done above. This gives a set of objects that are comparable within the whole sample volume, where the density level $D_{n}$ can be selected according to the properties of superclusters one wishes to study. As an example, for identifying structures, low density levels are better, but for studying the details of the structure, higher levels are useful; and sometimes it is necessary to use a set of luminosity levels. Examples include the density level 5.0 used by Einasto et al. (2011), level 4.6 used by Einasto et al. (2007), 5.5 in Luparello et al. (2011), and the set of levels in Lietzen et al. (2009). However, this approach is susceptible to Poisson noise, especially in sparser environments. It also does not take the richness differences of superclusters into account. We demonstrate both effects in Sect. 4.3.

Because of that, we offer an alternative procedure that assigns an individual threshold to each supercluster, adapting to the local density level. The idea is to follow the growth of individual superclusters from a compact volume around its centre, by lowering the density level and observing the supercluster mergers. By defining a supercluster as the volume within the density contour until the first merger, we can break the large-scale structure into a collection of compact components. Every component (supercluster) then has its own limiting density level $D_{\text {scl }}$, as is usual for other astronomical objects. We do not define galaxies by a common limiting stellar density level. As a result, we get a set of superclusters that forms the connected large-scale cosmic web.

To identify such superclusters in practise, it is easier to begin from lower densities and to proceed upwards. The mergers can now be seen as breakups of structures. We trace the splitting events in the density field objects tree. With a split a lower density filament ceases to be a "bridge" between two higher density regions. We pick the density value just above of the bridge, after the split, as the defining density level for these two objects. If one of these objects is broken up again at some higher threshold, it will not affect the other one.

As a downside this technique still requires manually setting several limits. First, the minimal size of a supercluster must be selected, for obviously some of the breaks involve objects that are too small to be of interest. In previous studies, a $100\left(h^{-1} \mathrm{Mpc}\right)^{3}$ lower volume limit was used by Einasto et al. (2007), Costa-Duarte et al. (2011) use ten galaxies as a minimum for their superclusters (in combination with the volume limit of $\left.64\left(h^{-1} \mathrm{Mpc}\right)^{3}\right)$. Luparello et al. (2011) use the object luminosity of $10^{12} L_{\odot}$ as the lower limit. In this study we use the diameter of the supercluster.

We must also choose the maximum threshold $D_{\text {lim }}$. While we observe that most of the superclusters are defined at similar density levels, some very rich clusters with their surroundings can satisfy the minimum size condition at a much higher level and the algorithm may break up well-established structures (we 
discuss these differences in Sect. 4.3). Because of that, we proceed in two steps. First we find the thresholds for all objects, and we find the maximum threshold $D_{\text {lim }}$ for superclusters as the density level where $95 \%$ of objects have a lower threshold. Then we recalculate the thresholds but prohibit splitting of structures above that threshold.

The natural lower density limit is the percolation density level. Percolation happens when the largest structure starts to fill the sample volume. In practise we define the percolation level $D_{\text {perc }}$ as the density when the richness of the second richest structure starts to decrease when lowering the density level (Martínez \& Saar 2002).

Shifting the maximum density threshold upwards will fragment structures further. Reducing the minimum size of a supercluster will have the same effect and will also increase the number of small objects.

We present the SDSS main and LRG supercluster catalogues in two versions, one with a set of fixed levels and the second with adaptive density thresholds. We describe the differences of these catalogues in more detail in Sect. 4.3.

\subsection{Supercluster properties}

After delineating superclusters as described in previous sections, we calculate a number of supercluster properties for all density levels using both the density field and the galaxy data. In the following we describe the calculation of the most important attributes of superclusters that will be included in the catalogues. The initial density peak, from which the supercluster grew and which usually indicates the presence of a large galaxy cluster, marks the supercluster position.

The supercluster volume is found from the density field as the number of connected grid cells multiplied by the cell volume:

$V_{\mathrm{scl}}=N_{\text {cellescl }} \Delta^{3}$,

where $\Delta$ is the grid cell length. We find also the sum of normalised densities at the grid vertices within the supercluster

$L_{\mathrm{scl}, \mathrm{df}}=\sum_{i \in \mathrm{scl}} \ell_{i} \cdot \Delta^{3}$,

for an estimate of the total luminosity of the supercluster.

Using galaxy luminosities, we obtain two more estimates for the total luminosity of the supercluster, the sum of the observed galaxy luminosities, and the sum of the weighted galaxy luminosities:

$$
\begin{aligned}
& L_{\mathrm{scl}, \mathrm{gal}}=\sum_{\mathrm{gal} \in \mathrm{scl}} L_{\mathrm{gal}}, \\
& L_{\mathrm{scl}, \mathrm{wgal}}=\sum_{\mathrm{gal} \in \mathrm{scl}} L_{\mathrm{gal}, \mathrm{w}} .
\end{aligned}
$$

We find the number of galaxies, $N_{\text {gal }}$ and, if available, the number of galaxy groups and clusters $N_{\mathrm{gr}}$ in a supercluster. We define the supercluster diameter $\varnothing$ as the maximum distance between its galaxies. Using the galaxy locations and their weighted luminosities, we find the supercluster centre of mass (luminosity):

$\boldsymbol{r}_{\mathrm{scl}}=\frac{1}{L_{\mathrm{scl}, \mathrm{wgal}}} \sum_{\mathrm{gal} \in \mathrm{scl}} \boldsymbol{r}_{\mathrm{gal}} \cdot W_{L}\left(d_{\mathrm{gal}}\right) L_{\mathrm{gal}}$.

Among these quantities, the most important are the supercluster diameter and the weighted total luminosity, because they are

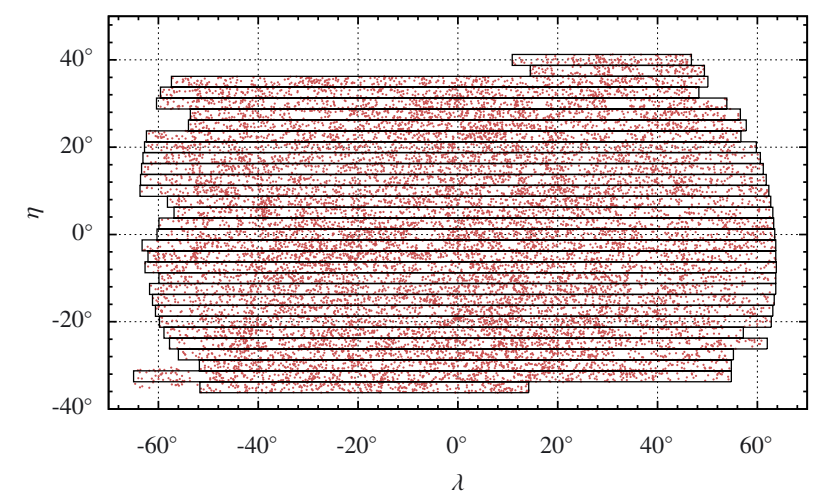

Fig. 1. The sky projection of the DR7 galaxies and the mask in the $\operatorname{SDSS} \eta$ and $\lambda$ survey coordinates.

least affected by the distance to the supercluster. We assume we have restored the real total luminosities by weighting the galaxies, and while we may lose dim galaxies, the brighter ones still mark the supercluster region sufficiently (Tempel 2011). Also, as we show later, neither of these properties is very sensitive to the choice of the density threshold. Because of the different weighting in the LRG sample, the weighted luminosity there cannot be used as an approximation to the total luminosity, as for the main sample.

We identify a supercluster by a "marker galaxy" that we arbitrarily choose to be a bright galaxy near the highest density peak in the supercluster volume. The aim of this is to tie a supercluster to an observational object and to construct an identifier that is not specific to the current catalogue. The long identification number is given in the format of $A A A \pm B B B+C C C C$, where $A A A$ and $B B B$ are the integer parts of the equatorial coordinates $\alpha$ and $\delta$ of the marker galaxy and $C C C C$, its redshift multiplied by 1000 .

We check whether a supercluster is in contact with the mask edge. A location near the sample boundary implies incompleteness of the supercluster, and its parameters may not reliable. Using the signal-to-noise field $G$ (Eq. (10), see also Appendix B), we calculate for each supercluster a confidence estimate

$C_{\mathrm{scl}}=\frac{1}{N_{\mathrm{gal}}} \sum_{\mathrm{gal} \in \mathrm{scl}} G\left(\boldsymbol{r}_{\mathrm{gal}}\right)$.

We interpolate the signal-to-noise ratio values of the density estimate to the galaxy locations and find the average over all galaxies in the supercluster. An extended description of supercluster properties in the catalogue is given in Appendix C.

\section{Galaxy and group data}

We constructed catalogues for both the SDSS main and LRG samples. The main sample has a high spatial density and allows to follow the superclusters in detail, but the LRG sample, although sparse, is much deeper.

\subsection{The SDSS main sample}

Our main galaxy sample is the main sample from the 7th data release of the Sloan Digital Sky Survey (Abazajian et al. 2009). We used the data from the contiguous 7646 square degree area in the North Galactic Cap, the so-called Legacy Survey (Fig. 1). The sample selection is described in detail in the SDSS DR7 group 
catalogue paper by Tago et al. (2010). We used galaxies with the apparent $r$ magnitudes $12.5 \leq m_{r} \leq 17.77$ and excluded duplicate entries. We corrected the redshifts of galaxies for the motion relative to the $\mathrm{CMB}$ and computed comoving distances of galaxies using the standard cosmological parameters: the Hubble parameter $H_{0}=100 \mathrm{hm} \mathrm{s}^{-1} \mathrm{Mpc}^{-1}$, the matter density parameter $\Omega_{\mathrm{m}}=0.27$, and the dark energy density parameter $\Omega_{\Lambda}=0.73$.

We calculated the absolute magnitudes of galaxies in the $r$ band as $M_{r}=m_{r}-25-\log _{10} d_{L}-K$, where $m_{r}$ is the Galactic extinction corrected apparent magnitude, $d_{L}=d(1+z)$ is the luminosity distance ( $d$ is the comoving distance) in $h^{-1} \mathrm{Mpc}$ and $z$ the redshift, and $K$ is the $k+e$ correction. The $k$-correction for the SDSS galaxies was calculated using the KCORRECT algorithm (Blanton et al. 2003a; Blanton \& Roweis 2007). In addition, we corrected the magnitudes for evolution, using the luminosity evolution model of Blanton et al. (2003b). The magnitudes correspond to the restframe (at the redshift $z=0$ ).

Groups and clusters of galaxies were determined using a modified friends-of-friends algorithm, in which a galaxy belongs to a group of galaxies if this galaxy has at least one group member galaxy closer than the linking length. To take selection effects into account when constructing a group catalogue from a flux-limited sample, we increased the linking length with distance, calibrating the scaling by shifting nearby groups (see Tago et al. 2010, for details). As a result, the sizes and velocity dispersions of our groups are similar at all distances. Our SDSS main galaxy sample contains 583362 galaxies and 78800 galaxy groups and clusters.

For the main sample, we use the apparent magnitude limits $m_{1}=12.5$ and $m_{2}=17.7$ for the luminosity limits $L_{1,2}$ in Eq. (4), and calculate the distance-dependent weight. We take $M_{\odot}=4.64$ mag in the $r$-band (Blanton \& Roweis 2007) as the luminosity of the Sun. For the luminosity function (Eq. (5)) we use the following parameter values: $\alpha=-1.42$ is the exponent at low luminosities $\left(L / L^{*}\right) \ll 1, \delta=-8.27$ is the exponent at high luminosities $\left(L / L^{*}\right) \gg 1, \gamma=1.92$ is a parameter that determines the speed of the transition between the two power laws, and $L^{*}$ (corresponds to $M^{*}=-21.97$ ) is the characteristic luminosity of the transition (Tempel et al. 2011). Figure 2 shows the dependence of the galaxy number density, the observed luminosity density, and the weighted luminosity density of galaxies on distance. Our weighting procedure has adequately restored total luminosities, the luminosity density does not depend on distance. The luminosity weights are shown in Fig. 3.

\subsection{The SDSS LRG sample}

The galaxies for the LRG sample were selected from the SDSS database by an SQL query requiring that the PrimTarget field should be either TARGET_GALAXY_RED or TARGET_GALAXY_RED_II. We demanded reliable redshifts (SpecClass $=2$ and $\mathrm{zConf}>0.95)$. We kept the galaxies within the same mask as the main galaxies (the compact continuous area in the Northern Galactic Cap). We calculated the absolute $M_{\mathrm{g}}^{\star}(z=0)$ magnitudes for the LRGs as in Eisenstein et al. (2001). We examined the photometric errors of the LRGs and deleted the galaxies brighter than $M_{\mathrm{g}}^{\star}=-23.4$ from the sample to keep the magnitude errors small. In total, our sample includes 170423 LRGs up to the redshift $z=0.6$ (the $k+e$-correction table in Eisenstein et al. 2001 stops at this redshift). It is worth mentioning that the LRG sample is approximately volume-limited (its number density is almost constant) between the distances from $400 h^{-1} \mathrm{Mpc}$ to $1000 h^{-1} \mathrm{Mpc}$.
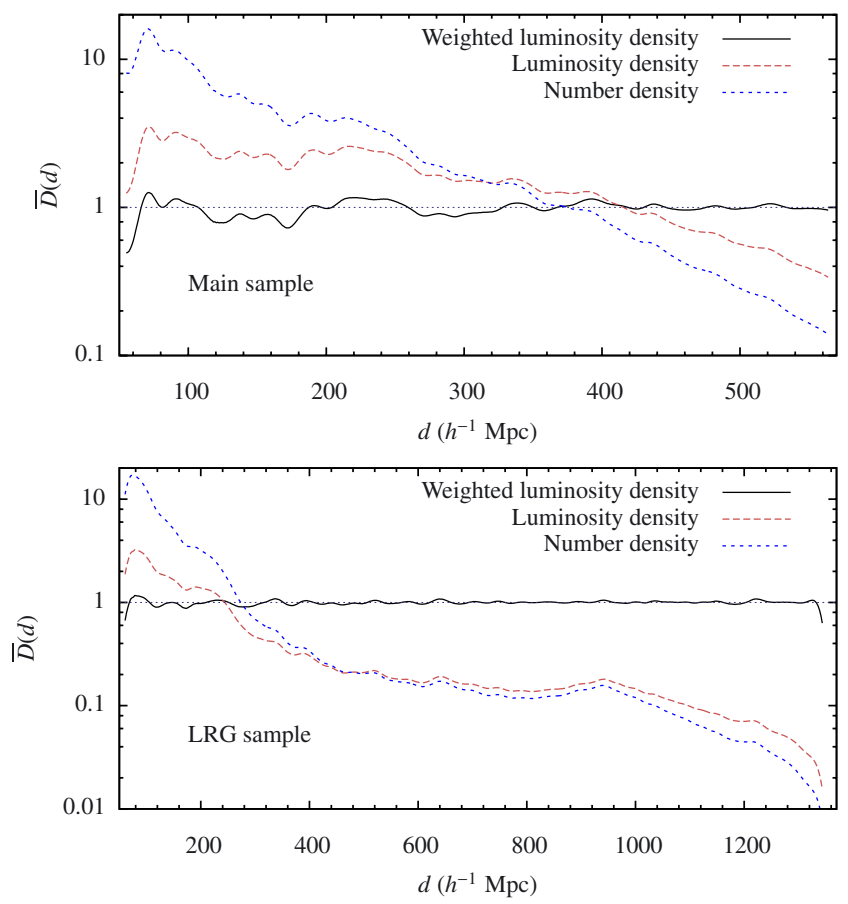

Fig. 2. Average normalised densities vs distance for the main (upper panel) and LRG samples (lower panel). The densities are averaged over thin (a few $h^{-1} \mathrm{Mpc}$ ) concentric shells of the distance $d$. Solid line the weighted luminosity density; dashed line - the observed luminosity density; dotted line - the galaxy number density.

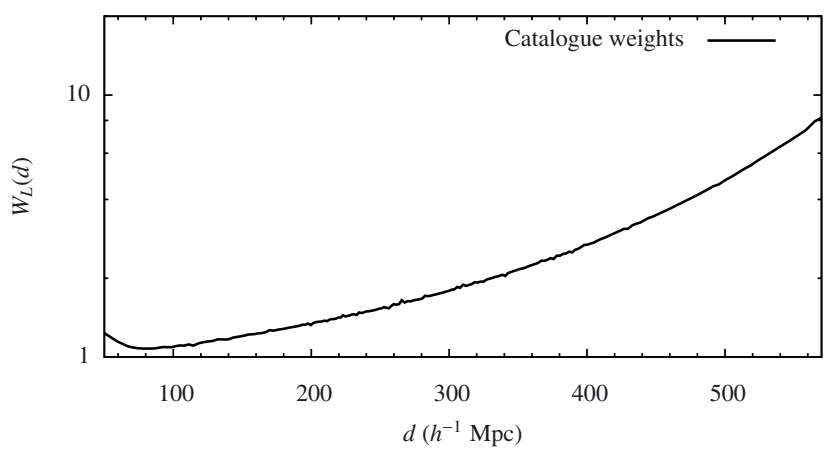

Fig. 3. Distance-dependent weights for the main sample galaxies.

We fix the fiducial comoving distance $d_{0}$ at $435.6 h^{-1} \mathrm{Mpc}$ $\left(z_{0}=0.15\right)$. The galaxies closer than that are fainter and are "not officially" LRGs (Eisenstein et al. 2001). By many properties they are yet similar to LRGs and we need these to be able to compare the main and LRG superclusters in the volume where the two galaxy samples overlap.

\subsection{The Millennium galaxy catalogue}

We chose a catalogue by Bower et al. (2006) that is an implementation of the Durham semi-analytic galaxy formation model on the Millennium Simulation by the Virgo Consortium (Springel et al. 2005). The catalogue is available from the Millennium database at the German Astrophysical Virtual Observatory $^{1}$. A subsample of about one million galaxies was

1 http://www.g-vo.org/www/Products/ MillenniumDatabases 
Table 1. Properties of the galaxy samples and of the density fields.

\begin{tabular}{lcccccccc}
\hline \hline Sample & $N_{\text {gal }}$ & $N_{\text {groups }}$ & $\begin{array}{c}\ell_{\text {mean }} \\
\frac{10^{10} h^{-2} L_{\odot}}{\left(h^{-1} \mathrm{Mpc}\right)^{3}}\end{array}$ & $\begin{array}{c}V_{\text {mask }} \\
\left(h^{-1} \mathrm{Gpc}\right)^{3}\end{array}$ & $\begin{array}{c}\Delta \\
h^{-1} \mathrm{Mpc}\end{array}$ & $\begin{array}{c}a \\
h^{-1} \mathrm{Mpc}\end{array}$ & $\begin{array}{c}d_{\min } \ldots d_{\max } \\
h^{-1} \mathrm{Mpc}\end{array}$ & $\begin{array}{c}z_{\min } \ldots z_{\max } \\
h^{-1} \mathrm{Mpc}\end{array}$ \\
\hline Main & 583362 & 78800 & $1.526 \times 10^{-2}$ & 0.132 & 1 & 8 & $55 \ldots 565$ & $0.02 \ldots 0.2$ \\
LRG & 170423 & - & $8.148 \times 10^{-4}$ & 1.789 & 2 & 16 & $60.5 \ldots 1346.4$ & $0.02 \ldots 0.5$ \\
Millennium & 1039919 & - & $1.304 \times 10^{-2}$ & 0.125 & 1 & 8 & - & - \\
\hline
\end{tabular}

Notes. $\ell_{\text {mean }}$ - mean density; $V_{\text {mask }}$ - sample mask volume; $\Delta$ - grid cell length; $a$-smoothing length; $d, z$ - distance and redshift limits.
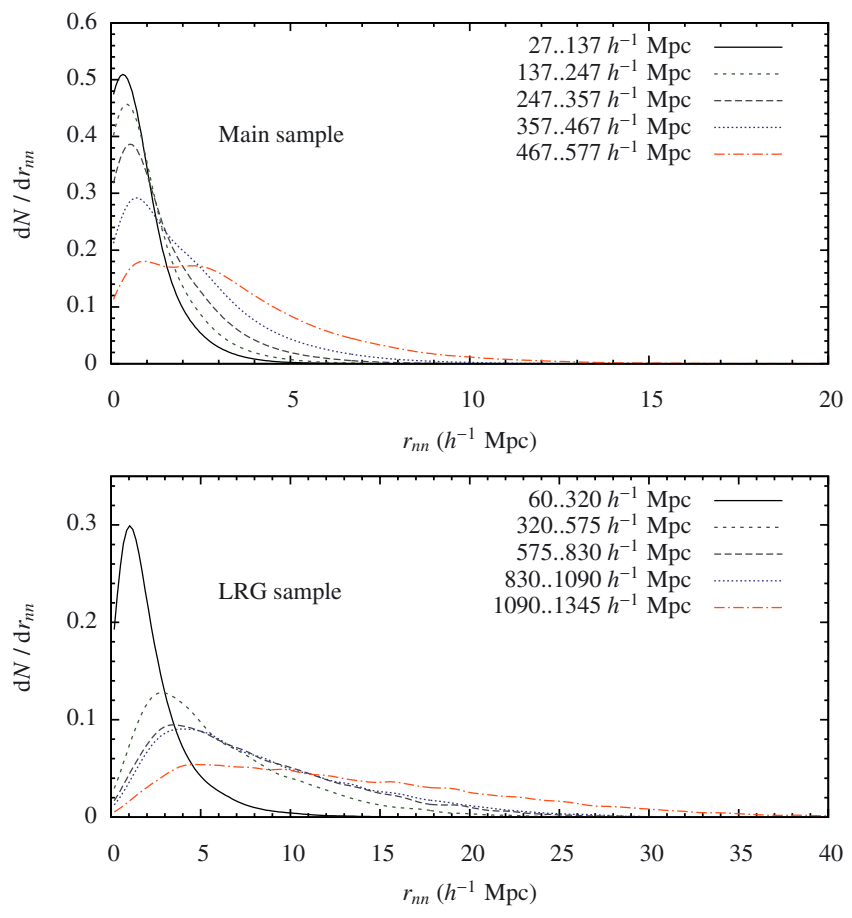

Fig. 4. The nearest neighbour distance distribution of the SDSS main (upper panel) and LRG sample (lower panel) galaxies. The distribution is shown for various distance intervals.

selected by the condition $M_{r}>-20.25$. This yielded a sample with almost the same number density of galaxies as that of the SDSS main sample (from 125 to $400 \mathrm{~h}^{-1} \mathrm{Mpc}$ ). We calculate the absolute luminosities for galaxies by taking $M_{\odot}=4.49$ and using the SDSS $r$ magnitudes (Vega) presented in the catalogue. This sample serves as a volume-limited test catalogue to study the performance of the supercluster finding algorithm.

\section{Density fields and superclusters}

\subsection{Density fields}

We chose the smoothing width of $a=8 h^{-1} \mathrm{Mpc}$ for the SDSS main sample. The choice of the kernel width is somewhat arbitrary, but an argument can be made that the scale has to correspond to the size of the structures we are searching for. For example, the kernel should be considerably wider than the diameters of galaxy clusters, which are a few megaparsecs. Also, we wish to be able to detect structures at large distances, where galaxies are sparser. We assume that the density field ties the galaxies together if these are separated by $2 a$. Figure 4 shows the nearest neighbour distributions for different distance intervals. We see that for the SDSS main sample the scale $a=8 h^{-1} \mathrm{Mpc}$ is comfortably large enough to group galaxies together even at far distances, and a slightly narrower kernel would also be sufficient. Historically, $a=8 h^{-1} \mathrm{Mpc}$ has been used in previous supercluster catalogues (Einasto et al. 2007) and in other supercluster studies (as in a more recent paper Costa-Duarte et al. 2011). As shown by Costa-Duarte et al. (2011), the density field method is actually not very sensitive to the choice of kernel width.

We first employ the sky projection mask (Fig. 1) used in Martínez et al. (2009) and then set the lower and higher limits for the distance. We do not need to use the more precise mask (e.g., the "mangle" mask provided by the NYU VAGC) because we are searching for structures of much larger dimensions. The angular diameter of the kernel at the far end of the sample is much larger (1.6 degrees for the main sample and 1.3 degrees for the LRGs) than these of the multitude of small holes inside the SDSS survey mask (with diameters less than an arcminute). The main sample density field mask is limited within the distances 55 to $565 h^{-1} \mathrm{Mpc}$. The distance limits here and also in case of the LRGs are chosen to avoid the distant incomplete regions.

We chose the kernel width for the SDSS LRG sample as $a=16 h^{-1} \mathrm{Mpc}$, twice the scale of kernel used for the main sample, since the LRG sample is sparser. Figure 4 demonstrates that most LRGs have at least one neighbour at distances up to $2 a=32 h^{-1} \mathrm{Mpc}$. The density field of the Millennium sample is calculated with $a=8 h^{-1} \mathrm{Mpc}$ kernel width. The mask is a cube with side length of $500 \mathrm{~h}^{-1} \mathrm{Mpc}$. Properties of the luminosity density fields for all three samples are given in Table 1.

\subsection{Uncertainty analysis}

Following the procedure described in Appendix B we created 100 realisations of both the main and LRG samples by randomly shifting galaxies. The shift scale was $8 h^{-1} \mathrm{Mpc}$ for the main and $16 h^{-1} \mathrm{Mpc}$ for the LRG sample. Figure 5 shows the dependence of $\sigma_{\ell}$ on distance. We can see the expected rise of $\sigma_{\ell}$ with a distance that is mainly caused by the decrease in the galaxy number density. We also see that the absolute values of the standard deviation are very low when compared to the density. This can be attributed to both the stability of the large-scale structures and the large smoothing scale for the density fields - several tens of galaxies contribute to the density at any point. Example maps of the density, standard deviation, and signal-to-noise fields spatial slices of the main sample are shown in Fig. 6. Looking at the images of the standard deviation field and the signal-to-noise field we can relate them to the observed large-scale structure. Nearby peaks in the density field stand out also in the signal-to-noise map, but the distant peaks already drown in the noise.

\subsection{Properties of superclusters}

\subsubsection{Superclusters of the main and LRG samples}

In this section we describe the general properties of the superclusters and also compare the fixed and adaptive threshold 

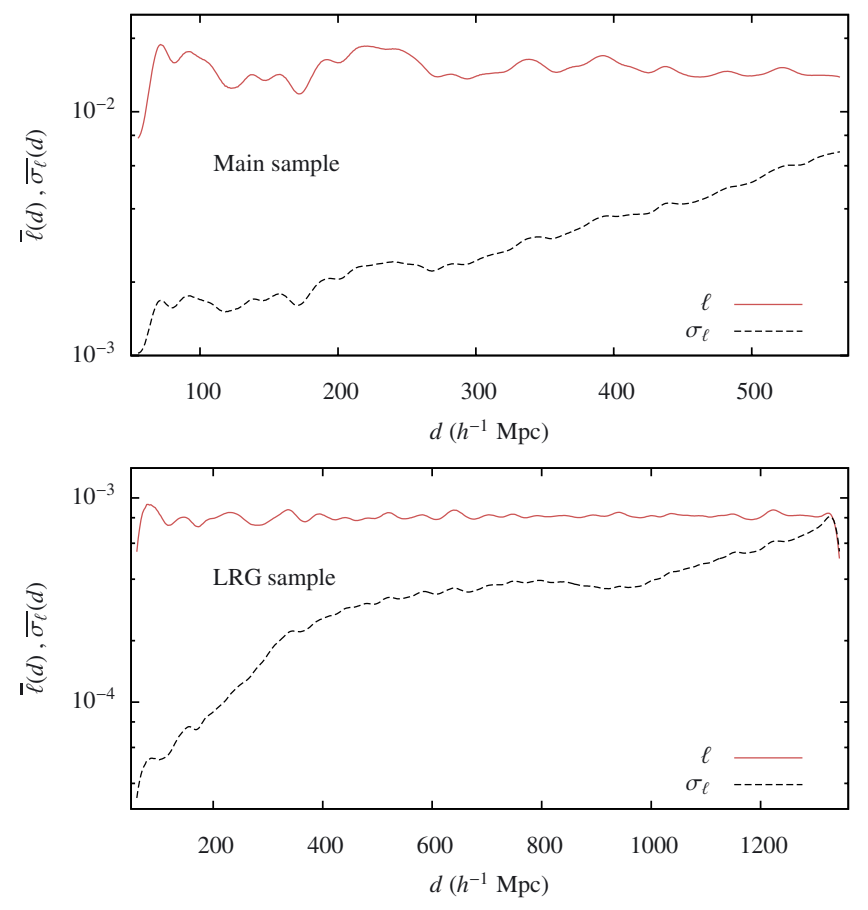

Fig. 5. The distance dependence of the average density and of the standard deviations for the main (upper panel) and LRG samples (lower panel).

catalogues. We chose the density difference between the thresholds as $\delta D=0.1$ (in the units of the mean density). We compare the diameters and the total weighted luminosities. At this stage we do not limit our object sample in any way - it also contains small objects that may consist of only one galaxy and superclusters located close to the boundary.

Figure 7 shows the diameter and weighted luminosity distributions for the main and LRG samples for different density levels. The shapes of the curves are similar in both samples, with the diameter distribution offering a slightly clearer picture. For all density thresholds, the maximum of the curves is located at about the same diameter/luminosity value. The slight dip in the distributions at small and dim objects is caused by our not including any density field objects that have no galaxies inside. At the high diameter/luminosity wings, the distributions have a series of maxima, which are characteristic of all density thresholds. This is caused by structures that are distinctively larger than most of the objects, and they are present even at high density levels $(D=8.0)$. As we move towards lower density levels $(D=6.0)$, the number of objects increases, while the objects themselves also get larger. The maxima caused by very large superclusters also become more prominent and at some point separate from the main body of the distribution, as they begin to include increasing numbers of smaller objects $(D=4.0)$. At the lowest density threshold, below percolation, there is one enormous structure that extends throughout the sample volume (at $D=2.0)$.

In Fig. 7 the distributions for the adaptive catalogues start at the minimum distance limit. They have higher values than the distributions for the fixed level superclusters because they include contributions from superclusters at several density thresholds.

Figure 8 presents an example of how the superclusters are affected by the two selection methods, fixed or adaptive density thresholds. The most noticeable consequence is that the superclusters $\mathrm{SCl} 64$ and $\mathrm{SCl} 94$ in the upper panel (a fixed density level) have both been broken in two and all their components have thresholds higher than before, while the superclusters $\mathrm{SCl} 1,362,578$ have been defined at lower density levels and $\mathrm{SCl} 1310$ has been assigned a much lower density level and is considerably larger because of that. The supercluster SCl 1320 does not meet the minimum diameter criterion $(\varnothing \geq$ $16 h^{-1} \mathrm{Mpc}$ ) and is not included in the catalogue with adaptive thresholds. If an object fails to qualify as a supercluster, it does not necessarily mean that galaxies belonging to this object are absent from the catalogue; instead of that, they can belong to some other supercluster at a lower threshold. This depends on the specific geometry of the supercluster environment.

We have to check whether the supercluster properties depend on distance and how the different density level assignments work. Figure 9 shows the dependence of the diameter and the weighted luminosity on distance. The extent of the scatter of diameters and luminosities increases with distance, while the averages remain more or less stable, and the standard deviations also do not exhibit systematic increase or decrease with the distance. The average diameter is almost constant for both the main and LRG samples. The barely noticeable downward trend in the fixed threshold supercluster catalogue is caused by small galaxy groups or even single galaxies, which are bright but do not form larger structures because of the sparseness of the galaxy sample. The weighted luminosity, however, tends to rise slightly for the main sample, and in a quite obvious manner for the LRG sample. Together, these graphs suggest that superclusters with similar dimensions are brighter at large distances, which implies some overweighting.

Figure 10 shows the dependence of the supercluster confidence estimates on supercluster richness (its number of galaxies) and distance. The confidence estimates are calculated as in Eq. (B.2). Both graphs display the expected behaviour. The confidence estimates diminish with distance, and richer superclusters also have higher signal-to-noise ratios. This property can be used to select objects for further studies. Predictably, the confidence estimates for superclusters in the LRG sample are significantly lower. The confidence estimates depend on the density threshold, but at lower density levels, more galaxies from the density field regions with higher variance are included. Because of that, fixed threshold superclusters have higher confidence estimates in Fig. 10.

Next we take a look at structure breakups and adaptively assigned supercluster thresholds. Figure 11 shows the number of splitting events, the percolation level, and the 95\% limiting density threshold for $D_{\text {scl }}$. Figure 12 gives an example of how supercluster diameters and luminosities change drastically during mergers when lowering the density level and, while still growing, remain relatively stable in between. Supercluster SCl 24 in Fig. 12 is a part of the Sloan Great Wall and at densities $D<4.7$ it actually includes all of the SGW superclusters (Einasto et al. 2010). For the main sample, density threshold assignments do not show any clear dependence on distance, while for the LRG sample the adaptively found levels are increasing with distance (Fig. 13). The broad peaks, which are visible in Figs. 9, 13, and 14 at approximately $250 \mathrm{~h}^{-1} \mathrm{Mpc}$ are caused by the Sloan Great Wall region superclusters.

Selection effects can cause the number density of superclusters to depend on distance. Figure 14 demonstrates that using a single density level to define superclusters causes a significant rise in the number of objects with distance. The reason for this is the Poisson noise that is caused by the increased density 

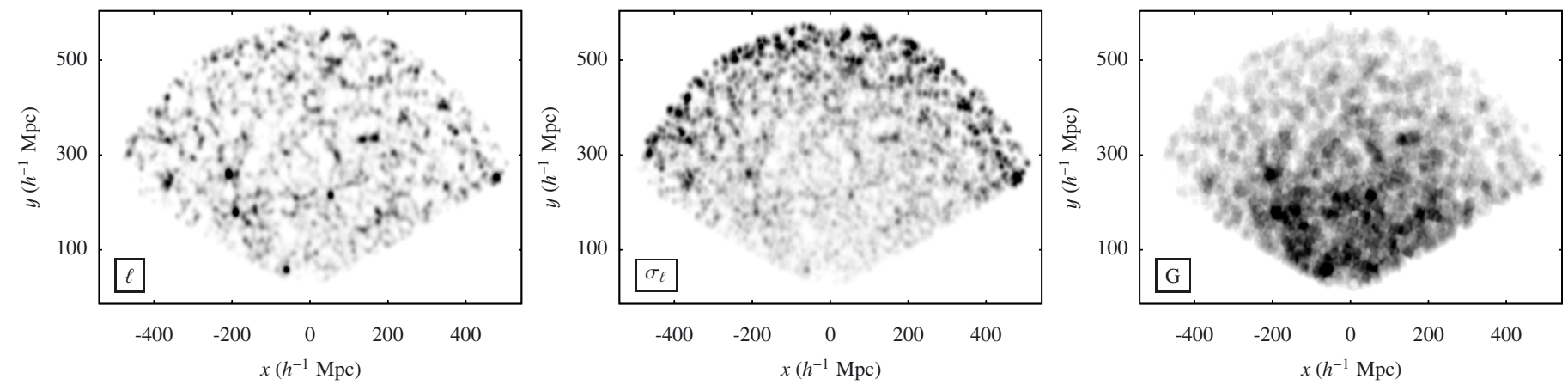

Fig. 6. A spatial slice of the luminosity density field for the main sample (left panel), the standard deviation field of the luminosity density (middle panel), and the signal-to-noise ratio field (right panel). The slice has a thickness of $1 h^{-1} \mathrm{Mpc}$ and is located at $z=33 h^{-1} \mathrm{Mpc}$ (Eq. (2)).
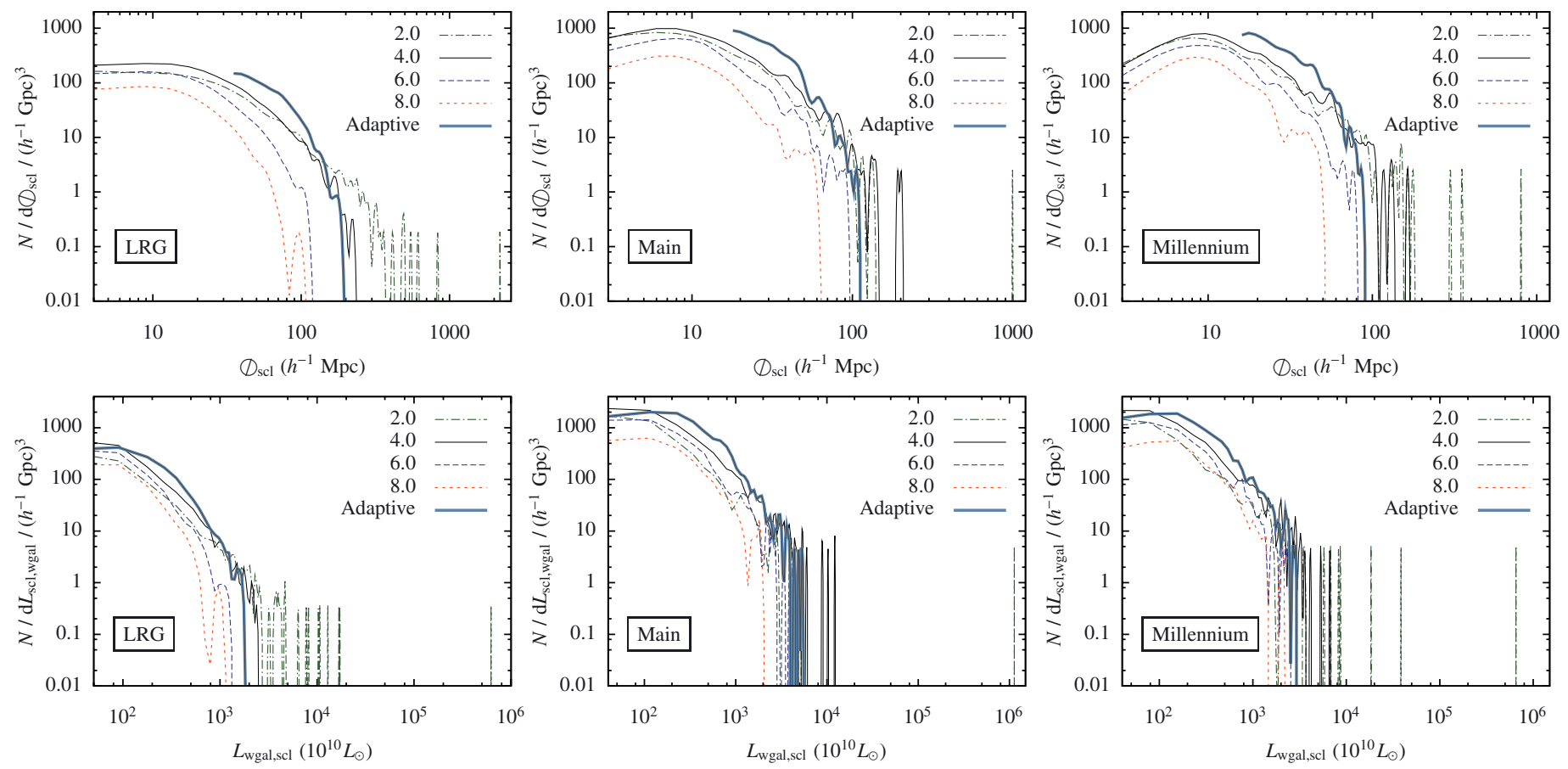

Fig. 7. The supercluster diameter (top row) and its weighted luminosity (bottom row) in the LRG, main, and Millennium samples (from left to right). Different lines correspond to different density thresholds.

contrast because of the weighting. As mentioned before, we can add the missing luminosity only where we see the galaxies, but not there where it is actually missing. In contrast, the number density of adaptive threshold catalogue superclusters is independent of distance.

\subsubsection{Superclusters of the Millennium sample}

We used the Millennium galaxy sample to evaluate the supercluster-finding procedure as applied to an ideal volumelimited sample. As a consequence, there are no distance-related effects. A separate further study will look more closely at the differences in object selection and their properties using several simulated flux and volume-limited samples.

Figure 7 allows us to compare the supercluster diameter and weighted luminosity distributions for the main sample to those for the Millennium sample. The distributions are strikingly similar, with the main sample containing almost the same amount of superclusters. The shape of the Millennium distributions at the density level $D=8.0$ also indicates the presence of large
Sloan Great Wall-like structures. Still, in contrast to the main sample, at the lowest density threshold there are also other large structures besides the one that has percolated. Also, the adaptive level superclusters appear to be slightly smaller than those in the main sample, which does not contradict earlier similar findings (Einasto et al. 2006). The number of structure splits versus the density threshold graphs for the Millennium and the main sample are virtually indistinguishable. They also share the percolation threshold and the $95 \%$ maximum density level differs by only one $\delta D$ (Fig. 11).

The summary of the properties of the supercluster catalogues for all three samples is given in Table 2. Both the main and LRG samples have most superclusters at the same threshold $D=3.0$, with 1566 and 4780 objects, accordingly (the volume of the LRG sample is about 14 times larger than that of the main sample). There is only one major difference with the Millennium sample: it has most superclusters, 1316 at a slightly higher threshold $D=3.3$, than the observational samples $(D=3.0)$. We find that significantly more galaxies belong to superclusters in the adaptive catalogue. For main and Millennium samples, the 

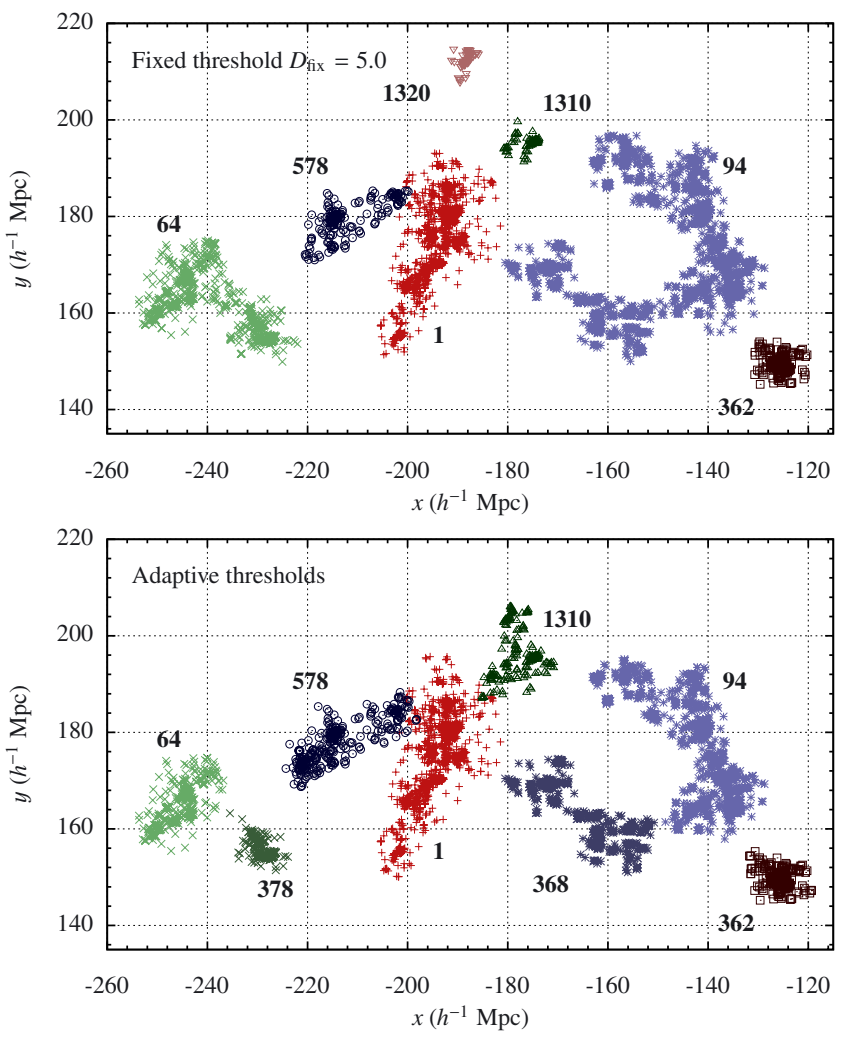

Fig. 8. The supercluster SCl 1 and the surrounding superclusters found with different methods for assigning the limiting threshold $D$. In the upper panel all superclusters have the same threshold $D_{\text {fix }}=5.0$. The adaptive density levels $D_{a}$ in lower panel are: $\mathrm{SCl} 1-D_{a}=4.7 ; \mathrm{SCl} 64$ and $\mathrm{SCl} 378-D_{a}=5.5 ; \mathrm{SCl} 94$ and SCl $368-D_{a}=5.3$; SCl $362-$ $D_{a}=4.4 ; \mathrm{SCl} 578-D_{a}=4.3 ; \mathrm{SCl} 1310-D_{a}=3.7$.

percentage rises from about $15 \%$ to more than a quarter of the galaxies, and in the LRG sample about $80 \%$ of the galaxies belong to superclusters in the adaptive threshold catalogue.

Comparison with the volume-limited Millennium sample shows that our supercluster algorithms generally work well and that we have avoided the selection problems inherent to magnitude-limited samples.

\subsubsection{Large-scale variations in the SDSS main sample}

If we look at the positions and density levels of the adaptivethreshold supercluster map of the SDSS main sample (Fig. 15), we see that there are strong variations in the supercluster thresholds depending on the region where they are located. The threshold level needed to define a supercluster is tightly correlated with the overall mean density. The spatial scale of these variations is about 200-300 $h^{-1} \mathrm{Mpc}$. One can discern the dominant supercluster plane (Einasto et al. 1997) and system of large voids behind it (described in detail by Platen 2009). The fact that these variations are not lost in the projection (we show the 2D projection of the full Legacy volume) shows that they are really huge. The reason for these variations is presently unclear, so we leave their quantification and study for the future.

\section{Conclusions and discussion}

Superclusters are the elements of the overall large-scale structure, the "LEGO pieces" of the Universe. As such, they describe the whole cosmic web of galaxies. They are also the largest objects of that web, and, although they are not gravitationally bound, in the future they may become bound isolated structures, the real "island Universes" (Araya-Melo et al. 2009).

Developing supercluster catalogues is useful for future, and sometimes unexpected, applications. The study of quasar environments (Lietzen et al. 2009) is a natural application of the multilevel supercluster catalogue, by aiding the uniform description of the overall matter density field. Searches for specific directions that are promising for observations is another example of where supercluster catalogues are indispensable; for example, a search for the elusive warm-hot intergalactic medium (WHIM) can be more effective with prior knowledge of the structures that are theoretically associated with the WHIM (Fang et al. 2010). And the identification of the Planck SZ source, mentioned in the introduction, is a perfect example of an unexpected development.

The main result of this work is a set of supercluster catalogues, based on the SDSS DR7 galaxy data, for the main and the LRG samples. The catalogues are public. We define superclusters, first for different mean density thresholds, and then for adaptive density thresholds that are different for each supercluster.

It is possible to create almost selection-free samples of superclusters from flux-limited catalogues. We studied the supercluster properties and found little dependence on the distance. We also compared the SDSS superclusters with the superclusters based on the Millennium galaxies, which were built using the same algorithms, and the supercluster samples have very similar properties.

While the LRG sample is very sparse and the number density of superclusters in its volume is much lower than for the main sample, one can still construct a supercluster sample with comparable properties.

When previous supercluster catalogues were based on fixed density levels (or nearest neighbour distances), we feel that the multiscaling (multi-threshold) approach is essential for defining the supercluster environment. The multi-level catalogues are useful for studying the overall density field, but for following individual superclusters, their structure, and their evolution, the adaptive threshold algorithm produces the best superclusters. With the full fixed threshold supercluster data set it is possible to create new adaptive threshold catalogues using alternative sets of limiting parameters. The adaptive threshold supercluster definition procedure permits more galaxies to be included in more superclusters, while also suppressing the selection effects. It allows us to generate practically volume-limited supercluster samples. In the LRG sample, the vast majority of galaxies are enclosed in superclusters. This is natural since LRGs are bright galaxies presumably residing in the cores of large galaxy groups, which in turn are very likely to be situated in superclusters (Einasto et al. 2003).

Galaxy superclusters are fairly well-defined systems. With the increasing density level, the supercluster sizes change radically with structure breaks, but are relatively stable in between, because they do not acquire or lose many galaxies while changing the density level. An important point is that at present, the number of known superclusters is small (especially the number of very large superclusters), which makes it possible to study them individually by looking at every one of them and correcting the possible glitches in their delineation.

There are certainly problems that remain unresolved at the moment. There is the question of boundary effects, for one using a fixed distance from the sample edge to limit the supercluster sample, as is sometimes done, is not entirely justified. First, it 

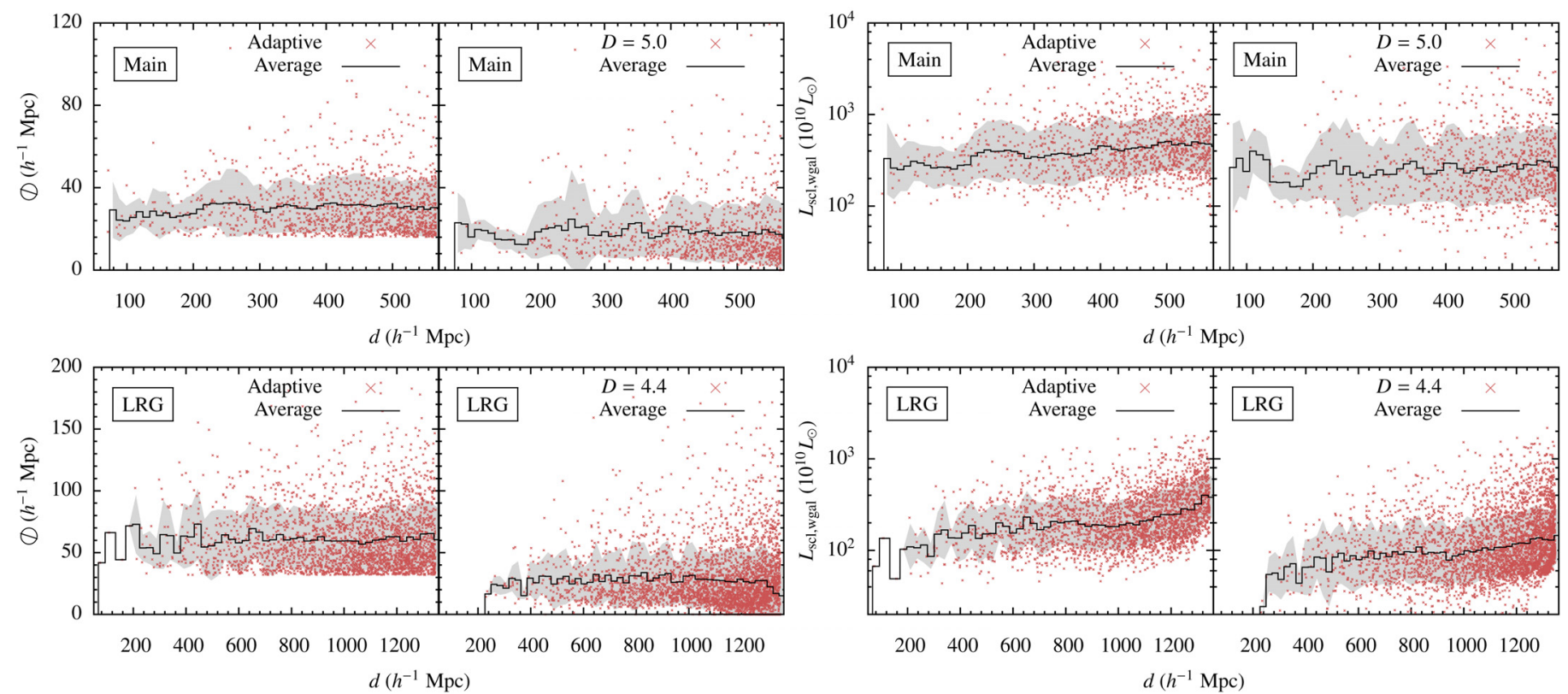

Fig. 9. Supercluster diameters (left panels) and total weighted luminosities (right panels) vs. distance for fixed and adaptive thresholds. The main sample is shown on the upper row and LRG on the lower. Points mark the diameters and luminosities of individual superclusters; the line is the average with the bin widths of $10 h^{-1} \mathrm{Mpc}$ for the main and $25 \mathrm{~h}^{-1} \mathrm{Mpc}$ for the LRG sample. The standard deviations in bins are shown with grey contours.

removes a large fraction of galaxies from the present samples. Second, many of the large superclusters (e.g., SCl 126 of the Sloan Great Wall, Einasto et al. 2001) are touching the SDSS mask edges, but are even so the largest between the superclusters. In fact, most of the nearby superclusters are incomplete because of the cone-like shape of the survey. Thus we also included such superclusters, and marked if those that were affected by the sample borders. It is already the decision of the catalogue users how they take that mark into account. Superclusters from the LRG sample show clear selection effects at the outer border of the sample volume. This is caused by the low number density and strong luminosity weighting. An unexpected result is that there is an overall density variation, and the variation of supercluster properties, on very large scales (about $200 \mathrm{~h}^{-1} \mathrm{Mpc}$ ), in the SDSS Legacy sample volume. We will discuss that in detail in the next paper.

Acknowledgements. The catalogue owes much to fruitful discussions with Vicent Martínez (Valencia) and with the members of the cosmology group at Tartu Observatory. Funding for the Sloan Digital Sky Survey (SDSS) and SDSS-II has come from the National Science Foundation, the US Department of Energy, the National Aeronautics and Space Administration, the Japanese Monbukagakusho, the Max Planck Society, and the Higher Education Funding Council for England. The SDSS Web site is http://www.sdss.org/. The SDSS is managed by the Astrophysical Research Consortium (ARC) for the Participating Institutions. The Participating Institutions include the American Museum of Natural History, Astrophysical Institute Potsdam, University of Basel, University of Cambridge, Case Western Reserve University, The University of Chicago, Drexel University, Fermilab, the Institute for Advanced Study, the Japan Participation Group, The Johns Hopkins University, the Joint Institute for Nuclear Astrophysics, the Kavli Institute for Particle Astrophysics and Cosmology, the Korean Scientist Group, the Chinese Academy of Sciences (LAMOST), Los Alamos National Laboratory, the Max-Planck-Institute for Astronomy (MPIA), the Max-Planck-Institute for Astrophysics (MPA), New Mexico State University, Ohio State University, University of Pittsburgh, University of Portsmouth, Princeton University, the United States Naval Observatory, and the University of Washington. The Millennium Simulation databases used in this paper and the web application providing online access to them were constructed as part of the activities of the German Astrophysical Virtual Observatory. We acknowledge the Estonian Science Foundation for support under grants 8005, 7765, and MJD272, the Estonian Ministry for Education and Science support by grants SF0060067s08, and also the support
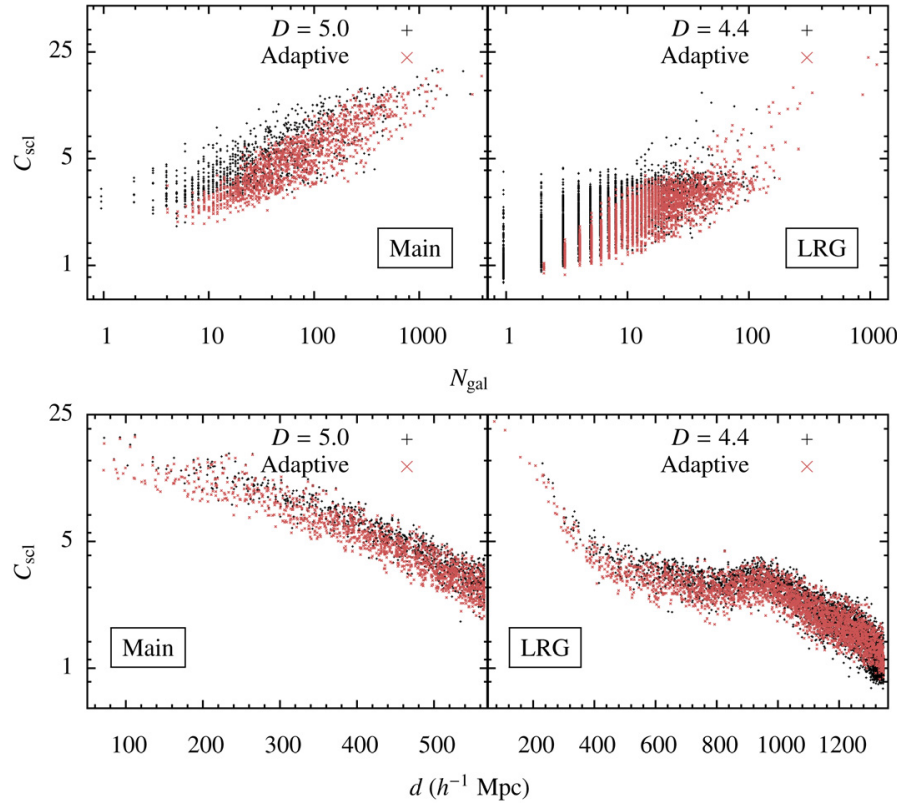

Fig. 10. Supercluster confidence estimates vs their richness (the number of member galaxies) (top panels) and vs their distance (bottom panels). The superclusters defined by a fixed threshold are marked with black, and those found using adaptive thresholds with red points.

by the Center of Excellence of Dark Matter in (Astro)particle Physics and Cosmology (TK120). Computations for the catalogues were carried out at the High Performance Computing Centre, University of Tartu.

\section{Appendix A: Kernel density estimates}

As superclusters are searched for as regions with the luminosity density over a certain threshold in a compact region of space, we have to convert the spatial positions of galaxies into a luminosity density field. The standard approach is to assume a Cox model 
L. J. Liivamägi et al.: SDSS DR7 superclusters
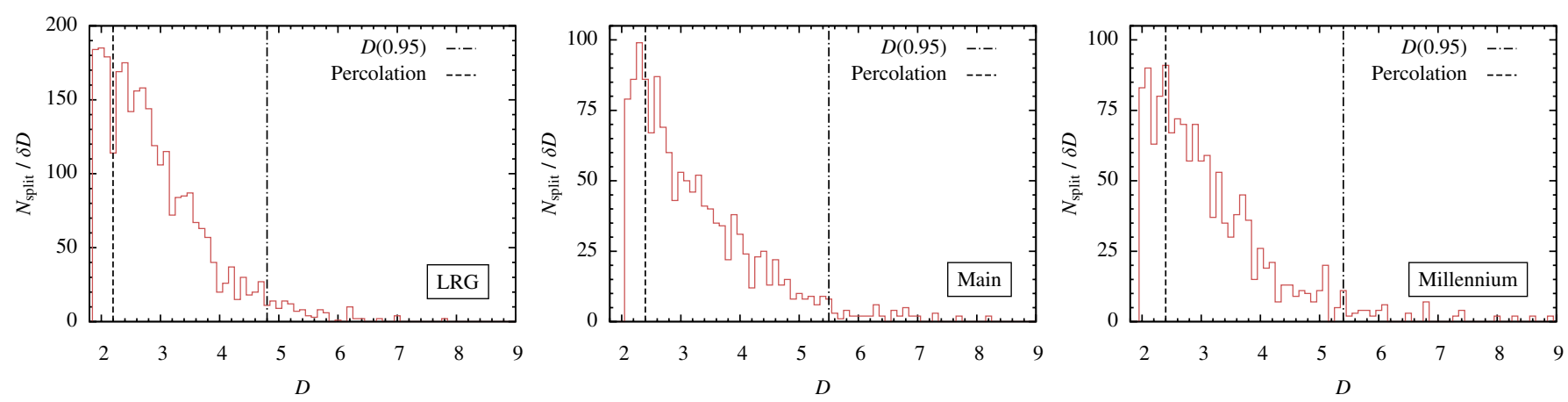

Fig. 11. The number of structure breaks (anti-mergers) per density threshold. Vertical lines denote the percolation and the upper limit densities. From the left: LRG, main, Millennium.

Table 2. Supercluster catalogue properties.

\begin{tabular}{|c|c|c|c|c|c|c|c|}
\hline $\begin{array}{l}(1) \\
\text { Sample }\end{array}$ & $\begin{array}{l}(2) \\
N_{\text {scl }}\end{array}$ & $\begin{array}{c}(3) \\
n_{\mathrm{scl}} \\
\left(h^{-1} \mathrm{Gpc}\right)^{-3}\end{array}$ & $\begin{array}{l}(4) \\
f_{\text {edge }}\end{array}$ & $\begin{array}{c}(5) \\
f_{\text {gal } \in \text { scl }}\end{array}$ & $\begin{array}{l}(6) \\
D_{\text {fix }} \\
\ell_{\text {mean }}\end{array}$ & $\begin{array}{c}(7) \\
D\left(N_{\max }\right) \\
\ell_{\text {mean }}\end{array}$ & $\begin{array}{c}(8) \\
n_{\mathrm{scl}}\left(D\left(N_{\max }\right)\right) \\
\left(h^{-1} \mathrm{Gpc}\right)^{-3}\end{array}$ \\
\hline Fixed threshold & & & & & & & \\
\hline Main & 982 & 7432 & 0.184 & 0.138 & 5.0 & 3.0 & 11852 \\
\hline LRG & 3761 & 2101 & 0.151 & 0.184 & 4.4 & 3.0 & 2671 \\
\hline Millennium & 844 & 6752 & 0.147 & 0.153 & 5.0 & 3.3 & 10528 \\
\hline (1) & (2) & (3) & (4) & $(8)$ & (9) & (10) & $(11)$ \\
\hline Sample & $N_{\text {scl }}$ & $\begin{array}{c}n_{\mathrm{scl}} \\
\left(h^{-1} \mathrm{Gpc}\right)^{-3}\end{array}$ & $f_{\text {edge }}$ & $f_{\mathrm{gal} \in \mathrm{scl}}$ & $\begin{array}{l}D_{\text {lim }} \\
\ell_{\text {mean }}\end{array}$ & $\begin{array}{l}D_{\text {perc }} \\
\ell_{\text {mean }}\end{array}$ & $\underset{h^{-1} \mathrm{Mpc}}{\bigcap_{\lim }}$ \\
\hline Adaptive threshold & & & & & & & \\
\hline Main & 1313 & 9937 & 0.225 & 0.267 & 5.5 & 2.4 & 16 \\
\hline LRG & 2701 & 1509 & 0.153 & 0.822 & 4.8 & 2.2 & 32 \\
\hline Millennium & 1214 & 9712 & 0.194 & 0.282 & 5.4 & 2.4 & 16 \\
\hline
\end{tabular}

Notes. Columns in Table: 1: sample name and threshold assigning method; 2: the number of superclusters; 3 : the number density of superclusters; 4: the fraction of superclusters close to the sample edge; 5: the fraction of galaxies in superclusters; 6: the fixed threshold value; 7: the density threshold with most objects; 8: the number density of objects for the threshold $D\left(N_{\max }\right)$; 9: the maximum allowed value for $D_{a} ; 10$ : the percolation threshold; 11: the minimum allowed supercluster diameter.

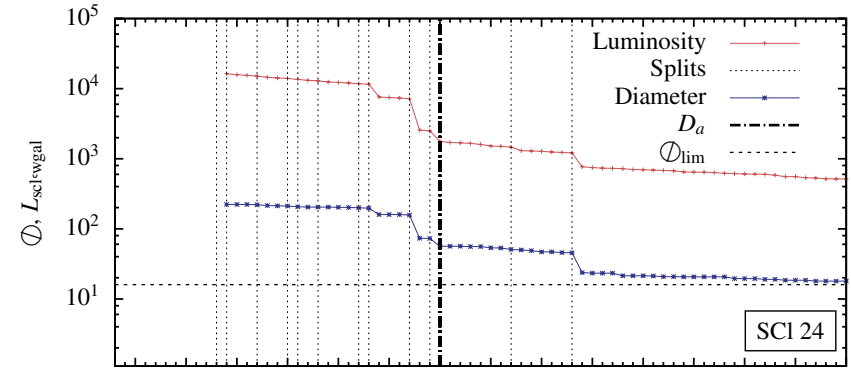

$\begin{array}{lllllllllllllll}2.0 & 2.5 & 3.0 & 3.5 & 4.0 & 4.5 & 5.0 & 5.5 & 6.0 & 6.5 & 7.0 & 7.5 & 8.0 & 8.5 & 9.0\end{array}$ $D$

Fig. 12. An example of the dependence of the supercluster diameter and the total luminosity on the density level $D$. Vertical thin dashed lines show splitting/merger events, and the thick dashed line shows the adaptive density threshold. The minimum diameter limit $16 h^{-1} \mathrm{Mpc}$ is also shown. The lines begin at the level where the object separates from the larger structure.

for the galaxy distribution, where the galaxies are distributed in space according to a inhomogeneous point process with the intensity $\rho(\boldsymbol{r})$ determined by an underlying random field (see, e.g. Martínez \& Saar 2002). The best way to estimate this intensity is by a kernel sum (Davison \& Hinkley 1997, Sect. 8.3.2):

$\rho(\boldsymbol{r})=\frac{1}{a^{3}} \sum_{i=1}^{N} K\left(\frac{\boldsymbol{r}-\boldsymbol{r}_{i}}{a}\right)$,

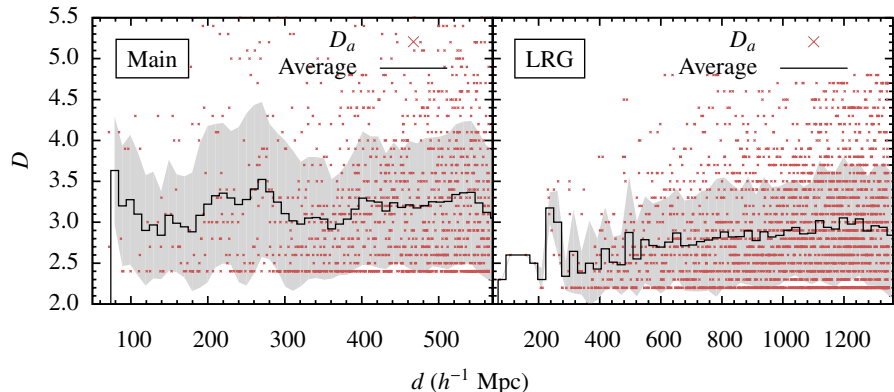

Fig. 13. Adaptively assigned supercluster thresholds, the average and the standard deviation vs. distance.

where the sum is over all $N$ data points, $\boldsymbol{r}_{i}$ are the coordinates, $K(\cdot)$ is the kernel, and $a$ the smoothing scale. As we estimate luminosities, we multiply kernel amplitudes by weighted galaxy luminosities $L_{\mathrm{gal}, \mathrm{w}}$ and calculate the luminosity density field as

$\ell(\boldsymbol{r})=\frac{1}{a^{3}} \sum_{\mathrm{gal}} K\left(\frac{\boldsymbol{r}-\boldsymbol{r}_{\mathrm{gal}}}{a}\right) L_{\mathrm{gal}, \mathrm{w}}$.

The kernels $K(\cdot)$ are required to be distributions, positive everywhere and integrating to unity; in our case,

$\int K(\mathbf{y}) \mathrm{d}^{3} y=1$ 

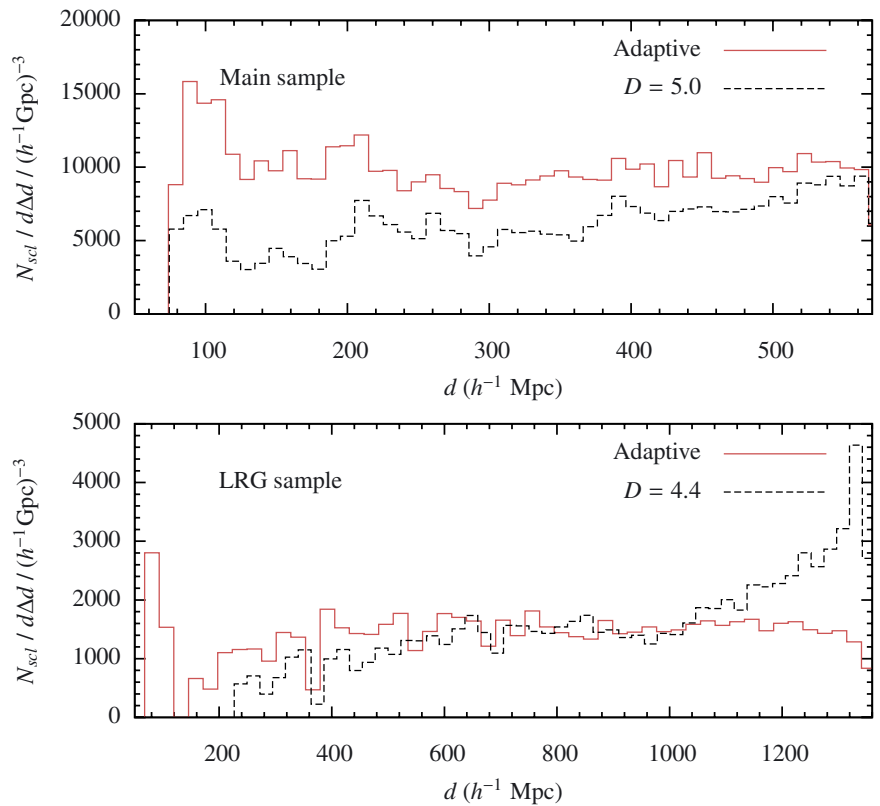

Fig. 14. The dependence of number densities on distance for adaptive and fixed threshold superclusters. The main sample is shown in the upper panel and the LRG sample in the lower panel.

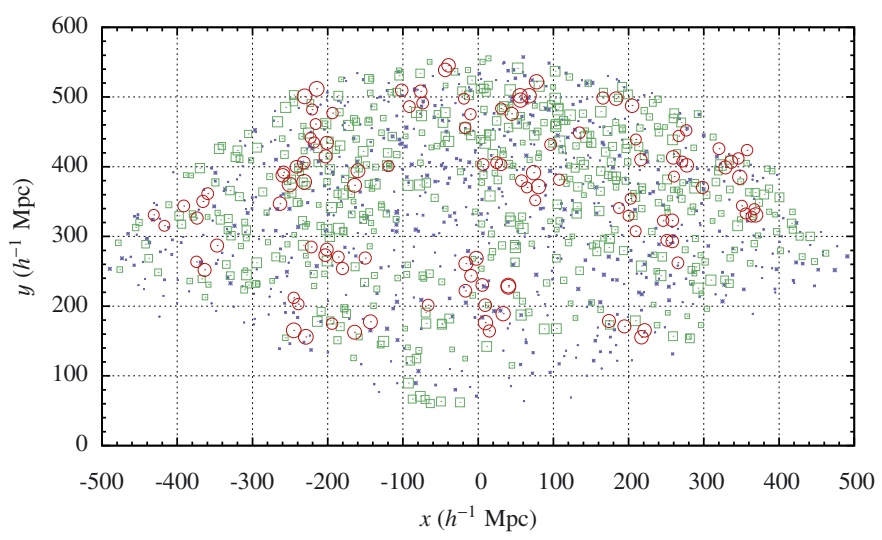

Fig. 15. The SDSS main sample supercluster map. Different symbol types and sizes show the density threshold levels used to delineate the superclusters; blue points: $D \leq 3.0$, green squares: $4.0<D \leq 4.5$, and red rings: $D>4.5$. The map is the $2 \mathrm{D}$ projection of the whole supercluster sample. Substantial differences in the levels can be seen e.g., in the regions around $(-60,300)$ and at the Sloan Great Wall region at $(0,220)$.

Good kernels for calculating densities on a spatial grid are the box splines $B_{J}$. They are local and they are interpolating on a grid:

$\sum_{i} B_{J}(x-i)=1$

for any $x$ and a small number of indices that give non-zero values for $B_{J}(x)$. To create our density fields we use the popular $B_{3}$ spline function:

$B_{3}(x)=\frac{|x-2|^{3}-4|x-1|^{3}+6|x|^{3}-4|x+1|^{3}+|x+2|^{3}}{12}$.

This function differs from zero only in the interval $x \in(-2,2)$, meaning that the sum in (A.4) only includes values of $B_{3}(x)$ at

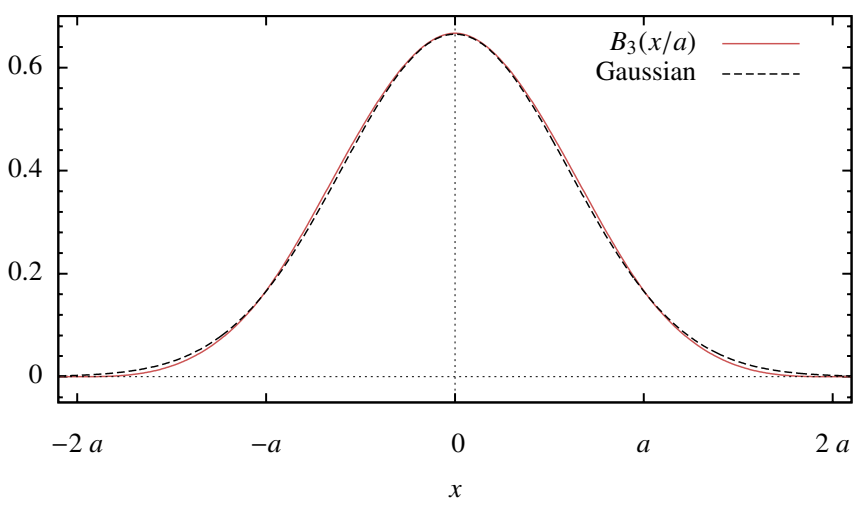

Fig. A.1. The shape of the kernel $B_{3}(x)$. Solid line - the $B_{3}(x)$ kernel; dashed line - a Gaussian with $\sigma=0.6$.

four consecutive arguments $x \in(-2,2)$ that differ by 1 . In practice, we calculate the kernel sum (A.3) on a grid. Let the grid step be $\Delta<a$, and $a=k \Delta$, where $k>1$ is an integer. Then the sum over the grid

$\sum_{i} B_{3}\left(\frac{x-i \Delta}{a}\right)=k$

because it consists of $k$ groups of four values of $B_{3}(\cdot)$ at consecutive arguments, differing by 1 . Thus, the kernel

$K_{B}^{(1)}(x / a ; \Delta)=\frac{\Delta}{a} B_{3}(x / a)$

differs from zero only in the interval $x \in[-2 a, 2 a]$ (Fig. A.1) and preserves the interpolation property exactly for all values of $a$ and $\Delta$, where the ratio $a / \Delta$ is an integer (also, the error is very small even if this ratio is not an integer, but $a$ is at least several times larger than $\Delta$ ). The three-dimensional kernel $K_{B}^{(3)}$ is given by a direct product of three one-dimensional kernels:

$$
\begin{aligned}
K_{B}^{(3)}(\boldsymbol{r} / a ; \Delta) & \equiv K_{B}^{(1)}(x / a ; \Delta) K_{B}^{(1)}(y / a ; \Delta) K_{B}^{(1)}(z / a ; \Delta) \\
& =\left(\frac{\Delta}{a}\right)^{3} B_{3}(x / a) B_{3}(y / a) B_{3}(z / a),
\end{aligned}
$$

where $\boldsymbol{r} \equiv\{x, y, z\}$. Although this is a direct product, it is practically isotropic (Saar 2009). This can be seen already from the fact that it is very close to a Gaussian with a mean zero and $\sigma=0.6$ (Fig. A.1), and the direct product of one-dimensional Gaussians is exactly isotropic.

\section{Appendix B: Error analysis of the density field}

To characterise the errors of our density field estimates we have to choose the statistical model for the galaxy distribution. The most popular model used for the statistics of the spatial distribution of galaxies in the Universe is the "Poisson model" (Peebles 1980), an inhomogeneous Poisson point process where the local intensity of the process is defined by the amplitude of the underlying realisation of a random field. In statistics it is called the Cox random process, see an introduction and examples in Martínez \& Saar (2002) and Illian et al. (1993). In cosmology, the random fields used are usually Gaussian or log-Gaussian fields.

As for any statistical model, it has been postulated to describe the galaxy distribution, and its success in applications describing the statistical properties of that distribution tends to support it. For example, this model was used to develop methods for 


\section{J. Liivamägi et al.: SDSS DR7 superclusters}

estimating the two-point correlation function (Hamilton 1993) and the power spectrum of the galaxy distribution (Tegmark et al. 1998). These methods have been extensively used to study the galaxy distribution. The same model serves as the basis for a maximum-likelihood approach to recover the large-scale cosmological density field by Kitaura et al. (2010).

We use the kernel method to estimate the intensity of our Cox process. A popular procedure for estimating the uncertainties of kernel-based intensity estimates for inhomogeneous Poisson processes is bootstrap (see, e.g., Davison \& Hinkley 1997, Sect. 8.3.2). Because the kernel used in estimating the intensity in Eq. A.1 is compact, there is only a finite and, in practice, a relatively small number of members in this sum. Bootstrap is used to estimate the sample errors (discreteness errors) caused by the discrete sampling. As stressed by Silverman \& Young (1987), bootstrap consists of two separate elements. Let our sample be $\left(X_{1}, \ldots, X_{n}\right)$. First, to estimate the discreteness error caused by the finite sample size $n$ of the sample parameter $\theta(X)$ that we are estimating, we use the sampling method, drawing a large number of samples of size $n$ from the (integral) population distribution function $F(X)$. Technically, it is the simplest method for generating random numbers with a given distribution - select $n$ uniform random numbers $U_{i}$ in the interval $(0,1)$ and select the sample value $X$ given by $F(X)=U$ for each $U$. The other element of bootstrap is to assume that the population distribution $F$ can be approximated by the empirical distribution function $F_{n}$ defined by all the $n$ observed values $\left(X_{1}, \ldots, X_{n}\right)$ that form the sample. If all $X_{i}$-s are i.i.d. (independent and identically distributed), this function can be defined as a step function with increments $1 / n$ at every $X_{j}^{\star}$, where $\left(X_{1}^{\star}, \ldots, X_{j}^{\star}\right)$ is an ordered growing sequence of the original sample values $X_{i}$. If we select from $F_{n}$, any bootstrap sample consists of the values of the original sample, selected from the original sample randomly with replacement (Efron \& Tibshirani 1993). Using the values of $\theta$ for all bootstrap samples, we can find the sampling errors (usually the bias and the variance) of the parameter $\theta$. These are the bootstrap error estimates we search for.

Theorems that prove the effectiveness of the bootstrap error estimates are usually proved for the case where both the sample size and the number of the bootstrap samples approach infinity (Shao \& Tu 1995), and for a finite sample size simulations are used. In our case, the sample that defines the intensity estimate for a given point in space consists of these galaxies, the positions of which are within the kernel volume around that point. The usual rule of thumb says that bootstrap error estimates may be considered reliable when the sample size is more than 30 (even sizes as small as 14 have been used in simulation examples, as in Efron \& Tibshirani 1993; Silverman \& Young 1987). Our kernel volumes include, on average, 150 galaxies in the case of the SDSS main sample and 25 galaxies for the LRG sample. At the density levels where we define our superclusters, $(D \approx 5)$, the corresponding numbers are 750 and 125 , so our error estimates should be reliable enough.

For the inhomogeneous Poisson process, where the points $\boldsymbol{X}_{i}$ are identically and independently distributed with the locally defined intensity $\lambda$, bootstrap can be used to estimate the errors of the kernel estimate (Eq. (A.1)). In practice, for intensity estimation a bootstrap version that is called a smoothed bootstrap is used. This is a version of parametric bootstrap, using, instead of the empirical distribution function, its smoothed version. Silverman \& Young (1987) demonstrated that it is more effective in estimating the variance of intensity as the standard bootstrap. To use that, in practice, we generate bootstrap samples of the same size as the original sample, selecting the galaxies from our sample randomly with replacement, as usual in bootstrap, but give the selected galaxies random displacements. As explained in Davison \& Hinkley (1997) and Silverman \& Young (1987), the random spatial displacements are required to have the probability density of the same form as the kernel function, but it is useful to undersmooth, using the kernel for the displacements that is narrower than the kernel used for calculating the intensity estimates. We undersmooth by a factor of two. As our grid size is huge (about $10^{8}$ ), we use 100 bootstrap samples for each grid vertex. This number has been found to be large enough to estimate the sample variance, based on simulation studies (Efron \& Tibshirani 1993).

Another point that has to be taken care of when estimating global (population) statistics as correlation functions or power spectra (spectral densities) of Cox processes is to account for the difference of the measured statistic for the specific realisation of the random field and for the random field as a whole (the so-called cosmic noise problem, see, e.g., Szapudi \& Colombi 1996; Peacock 1999, p. 522). The discreteness errors, estimated by bootstrap, and the realisation variance combine in a subtle way (Cohn 2006). In our case, fortunately, the spatial density - the intensity of the Cox process that we are estimating (the geography of the large-scale structure) is exactly the underlying random realisation itself - we are measuring the cosmic noise, so are not interested in the mean density of the Universe. The only errors our intensity estimates have are discreteness errors, and these can be estimated by bootstrap.

We select the galaxies for the bootstrap samples, together with their measured luminosities, and we consider galaxy distribution as a marked Cox process, with luminosities as marks. If we could statistically model the luminosity distribution among galaxies as random (a random marks model, see, e.g., Illian et al. 1993), we could build bootstrap samples by randomly relabelling galaxies, choosing their luminosities as in the usual bootstrap from the luminosities of the sample galaxies inside the kernel volume. This, however, would not be right, as galaxies are well known to be segregated by luminosity - more luminous galaxies populate regions of higher number density of galaxies (Hamilton 1988; Girardi et al. 2003). We chose another way and tried modelling the luminosity errors. These consist of a small error of the luminosity weights, generated by the errors of the luminosity function, and an error in modelling the evolution correction (Blanton et al. 2003b). We tested the effect of these errors by selecting them randomly from the observed distributions, compared the intensity estimates with modified luminosities and with fixed luminosities, and found no significant differences. As the luminosity errors were much smaller than the deviations of the intensity estimates generated by bootstrap, the discreteness errors, and we did not find a good statistical model to describe them, we ignored these errors.

After calculating the positions for the galaxies of a bootstrap sample, we find a new intensity estimate. We repeated the procedure a number of times (for this paper, we generated 100 bootstrap samples for every grid point where we estimated the intensity) and found the standard deviation for the intensity $\sigma_{\ell}$ for each grid vertex as

$$
\sigma_{\ell}=\sqrt{\frac{1}{N} \sum_{m=1}^{N}\left(\ell_{m}^{*}-\overline{\ell^{*}}\right)^{2}},
$$

where $N$ is the number of bootstrap realisations, $\ell_{m}^{*}$ the intensity for a bootstrapped sample, and $\overline{\ell^{*}}$ its mean over all realisations. 
We also found the "signal-to-noise ratio" for each grid point:

$G=\frac{\ell}{\sigma_{\ell}}$

\section{Appendix C: Description of the catalogue}

The catalogue consists of several tables with some redundancies between them. For each density level $D$ there exists a table with all superclusters found at that threshold. These tables contain the following information (some less important properties are omitted here, but can be found in the readme files):

- an unique identification number in the long and short forms;

- the number of galaxies and groups (the latter for the main sample alone);

- the supercluster volume as the number of the constituent grid cells times the cell volume (Eq. (12));

- the supercluster luminosity as the sum of densities at grid vertices (Eq. (13));

- the supercluster luminosity as the sum of the observed galaxy luminosities (Eq. (14));

- the supercluster luminosity as the sum of the weighted galaxy luminosities (Eq. (15)). For the main sample supercluster catalogue, we consider this as the best estimate of the total luminosity of the supercluster;

- the maximum density in the supercluster;

- the equatorial coordinates (J2000 here and hereafter) and the comoving distance of the highest density peak;

- the equatorial coordinates and the comoving distance of the centre of mass (Eq. (16));

- the Cartesian coordinates (Eq. (2)) of the highest peak and of the centre of mass;

- the supercluster diameter as the maximum distance between the galaxies in the supercluster;

- the identifier of the "marker" galaxy in the Tago et al. (2010) catalogue;

- the equatorial coordinates and the redshift of the "marker" galaxy;

- the confidence estimate for the supercluster found from the signal-to-noise field $G$ (Eq. (B.2));

- shows if a supercluster is in contact with the mask boundary (1- yes, 0 - no);

- the number of objects that will split from the supercluster above the current density threshold.

A similarly structured supercluster catalogue with adaptively assigned density thresholds has been compiled by combining the supercluster data in the tables described above. For each supercluster we take the data from the fixed level catalogue that corresponds to its defining density level and add the threshold value.

Additionally, we provide lists of galaxies and groups, together with the supercluster identifiers they are attributed to, for all density levels. We also present the supercluster splitting tree in the form of a table, where each supercluster is given the identifier of the object it belongs to at all given thresholds.

As the full volume of the supercluster catalogues is very large, we have chosen to upload only a part of them to the CDS. There are the two adaptive catalogues, one for the main sample and the other for the LRGs, and two fixed-level catalogues, of $D=5.0$ for the main sample and of $D=4.4$ for the LRGs. The full catalogue is accessible at: http://atmos. physic.ut.ee/ juhan/super/ with a complete description in the readme files.

\section{References}

Abazajian, K. N., Adelman-McCarthy, J. K., Agüeros, M. A., et al. 2009, ApJS, 182,543

Aragón-Calvo, M. A., van de Weygaert, R., \& Jones, B. J. T. 2010, MNRAS, 408, 2163

Araya-Melo, P. A., Reisenegger, A., Meza, A., et al. 2009, MNRAS, 399, 97

Basilakos, S. 2003, MNRAS, 344, 602

Blanton, M. R., \& Roweis, S. 2007, AJ, 133, 734

Blanton, M. R., Brinkmann, J., Csabai, I., et al. 2003a, AJ, 125, 2348

Blanton, M. R., Hogg, D. W., Bahcall, N. A., et al. 2003b, ApJ, 592, 819

Bower, R. G., Benson, A. J., Malbon, R., et al. 2006, MNRAS, 370, 645

Cohn, J. D. 2006, New Astron., 11, 226

Costa-Duarte, M. V., Sodré, Jr., L., \& Durret, F. 2011, MNRAS, 411, 1716

Davison, A. C., \& Hinkley, D. V. 1997, Bootstrap Methods and Their Application (Cambridge, UK: Cambridge University Press)

de Vaucouleurs, G. 1953, AJ, 58, 30

Einasto, M., Liivamägi, L. J., Tempel, E., et al. 2011, ApJ, 736, 51

Efron, B. \& Tibshirani, R. J. 1993, An introduction to the bootstrap (London, UK: Chapman and Hall)

Einasto, M., Tago, E., Jaaniste, J., Einasto, J., \& Andernach, H. 1997, A\&AS, 123,119

Einasto, M., Einasto, J., Tago, E., Müller, V., \& Andernach, H. 2001, AJ, 122, 2222

Einasto, J., Hütsi, G., Einasto, M., et al. 2003, A\&A, 405, 425

Einasto, J., Einasto, M., Saar, E., et al. 2006, A\&A, 459, L1

Einasto, J., Einasto, M., Tago, E., et al. 2007, A\&A, 462, 811

Einasto, M., Tago, E., Saar, E., et al. 2010, A\&A, 522, A92

Eisenstein, D. J., Annis, J., Gunn, J. E., et al. 2001, AJ, 122, 2267

Fang, T., Buote, D. A., Humphrey, P. J., et al. 2010, ApJ, 714, 1715

Girardi, M., Rigoni, E., Mardirossian, F., \& Mezzetti, M. 1993, ApJ, 417, 19

Girardi, M., Rigoni, E., Mardirossian, F., \& Mezzetti, M. 2003, A\&A, 406, 403

Hamilton, A. J. S. 1988, ApJ, 331, L59

Illian, J., Penttinen, A., Stoyan, H., \& Stoyan, D. 1993, Statistical Analysis and Modelling of Spatial Point Patterns (Chischester, UK: John Wiley \& Sons)

Jasche, J., Kitaura, F. S., Li, C., \& Enßlin, T. A. 2010, MNRAS, 409, 355

Kitaura, F.-S., Jasche, J., \& Metcalf, R. B. 2010, MNRAS, 403, 589

Lietzen, H., Heinämäki, P., Nurmi, P., et al. 2009, A\&A, 501, 145

Luparello, H., Lares, M., Lambas, D. G., \& Padilla, N. 2011, MNRAS, 415, 964

Martínez, V. J., \& Saar, E. 2002, Statistics of the Galaxy Distribution (Boca Raton: Chapman \& Hall/CRC)

Martínez, V. J., Arnalte-Mur, P., Saar, E., et al. 2009, ApJ, 696, L93

Peacock, J. A. 1999, Cosmological Physics (UK: Cambridge University Press)

Peebles, P. J. E. 1980, The large-scale structure of the universe (Princeton, US: Princeton University Press)

Planck Collaboration, Aghanim, N., Arnaud, M., et al. 2011, A\&A, 536, A9

Platen, E. 2009, Ph.D. Thesis, University of Groningen

Saar, E. 2009, in Data Analysis in Cosmology, ed. V. J. Martínez, E. Saar, E. Martínez-González, \& M.-J. Pons-Bordería, Lecture Notes in Physics (Berlin: Springer Verlag), 665, 523

Shao, J., \& Tu, D. 1995, The Jackknife and Bootstrap (Heidelberg, Germany: Springer-Verlag GmbH)

Silverman, B. W., \& Young, G. A. 1987, Biometrika, 74, 469

Springel, V., White, S. D. M., Jenkins, A., et al. 2005, Nature, 435, 629

Szapudi, I., \& Colombi, S. 1996, ApJ, 470, 131

Tago, E., Saar, E., Tempel, E., et al. 2010, A\&A, 514, A102

Tegmark, M., Hamilton, A. J. S., Strauss, M. A., Vogeley, M. S., \& Szalay, A. S. 1998, ApJ, 499, 555

Tempel, E. 2011, Ph.D. Thesis, University of Tartu

Tempel, E., Einasto, J., Einasto, M., Saar, E., \& Tago, E. 2009, A\&A, 495, 37

Tempel, E., Saar, E., Liivamägi, L. J., et al. 2011, A\&A, 529, A53

Wake, D. A., Nichol, R. C., Eisenstein, D. J., et al. 2006, MNRAS, 372, 537 